\title{
BECN Homologs And ATG14 Form A Conserved, Metastable Coiled-Coil To Mediate Autophagy
}

\author{
Sangita C. Sinha, Minfei Su, Yang Mei, Yue Li and Christopher L. Colbert.
}

Department of Chemistry and Biochemistry, North Dakota State University, Fargo, ND 58108-6050, USA.

Autophagy, an essential eukaryotic cellular homeostasis pathway, is responsible for sequestration of unwanted, damaged or harmful cytoplasmic components in vesicles called autophagosomes, followed by their lysosomal degradation, thereby enabling nutrient recycling. Autophagosome nucleation is mediated by Class III phosphatidylinositol 3-kinase complexes that include two key autophagy proteins, a BECN (also called Beclin, ATG6 or VPS30) homolog and ATG14/BARKOR. BECN1 was amongst the first mammalian autophagy proteins to be identified. Recently, mammals were shown to encode a second BECN paralog, BECN2. BECN2 shares $57 \%$ sequence identity with BECN1 is also involved in autophagy as well as in ligandinduced endolysosomal degradation of cellular and viral-encoded GPCRs. BECN2 heterozygous knockout mice have defective autophagy, increased levels of brain cannabinoid 1 receptor, elevated food intake, obesity and insulin resistance.

Here we focus on understanding the structure of the $\alpha$-helical, coiled-coil domain (CCD) of BECN homologs and the similarities and differences in their interaction with ATG14. X-ray crystal structures of the CCDs of human BECN1 and BECN2, show that they form similar autophagy-inactive, anti-parallel homodimers, which includes six and seven non-ideal packing interactions respectively, although the BECN2 CCD homodimer is significantly more curved and has a tighter binding affinity than that of BECN1. In contrast, SAXS analyses indicate that the CCDs of both BECN1 and BECN2 bind the ATG14 CCD to form a parallel, curved heterodimer stabilized by multiple, conserved polar interactions. Computational models of the ATG14:BECN CCD heterodimers fitted to SAXS envelopes enable identification of interface residues. Isothermal calorimetry experiments demonstrate that compared to BECN1, the BECN2 CCD forms a stronger heterodimer with the ATG14 CCD. Mutation of non-ideal BECN1/BECN2 interface residues to more ideal pairs improves homodimer selfassociation and thermal stability. Many of these BECN1 mutations knock-out binding to ATG14, but all BECN2 CCD mutants bind ATG14, although more weakly than wild-type. Thus, polar CCD interface residues of BECN homologs result in a metastable homodimer, facilitating dissociation; but enable better interactions with polar ATG14 residues stabilizing the BECN:ATG14 heterodimer. Further, these structure-based mechanistic differences in BECN1 and BECN2 homodimerization and heterodimerization likely dictate competitive ATG14 recruitment and enable a dynamic exchange between diverse CCD-containing partners within the cell. 